\title{
Experimental and clinicopathological analysis of HOXB9 in gastric cancer
}

\author{
FUMIHIKO KATO, NORIHITO WADA, TETSU HAYASHIDA, KAZUMASA FUKUDA, RIEKO NAKAMURA, \\ TSUNEHIRO TAKAHASHI, HIROFUMI KAWAKUBO, HIROYA TAKEUCHI and YUKO KITAGAWA
}

\author{
Department of Surgery, Keio University School of Medicine, Tokyo 160-8582, Japan
}

Received June 25, 2016; Accepted December 3, 2018

DOI: $10.3892 / 01.2019 .10008$

\begin{abstract}
The association between homeobox (HOX)B9 expression and tumor malignancy was identified recently. It was reported that HOXB9 induced tumor angiogenesis, and associated with poor prognosis in patients with breast and colon cancer. On the other hand, regional lymph nodes are the most common site of tumor spread, and lymph node metastasis is a major prognostic factor in gastric cancer. It was hypothesized that HOXB9 promotes tumor lymphangiogenesis and induces tumor progression, invasion and metastasis in gastric cancer. The aim of the present study was to evaluate the correlation between HOXB9 expression, prognosis and clinicopathologic factors in patients with gastric cancer, and to assess the contribution of HOXB9 expression to tumor cell lymphangiogenesis in vitro. HOXB9 expression was evaluated by immunohistochemistry in resected tumor tissues from 58 patients with gastric cancer, and the association between prognosis and clinicopathologic factors was determined. HOXB9 gene was overexpressed in human gastric cancer TMK-1 cells and the effect of HOXB9 overexpression on the expression of vascular endothelial growth factor (VEGF)-C, VEGF-D and VEGF receptor (R)-3 was determined. It was demonstrated that the depth of tumor invasion, the number of node metastases, lymphatic invasion and vascular invasion were significantly associated with HOXB9 expression. Overall survival was decreased in patients with HOXB9 expression. The mRNA expression of VEGF-D but not of VEGF-C and VEGFR-3 was increased in HOXB9-overexpressing TMK-1 cells compared with control cells. In conclusion, HOXB9 expression was positively correlated with gastric cancer progression and lymphangiogenesis marker expression. HOXB9 may be associated with lymphogenic metastasis.
\end{abstract}

Correspondence to: Dr Norihito Wada, Department of Surgery, Keio University School of Medicine, 35 Shinanomachi, Shinjuku-ku, Tokyo 160-8582, Japan

E-mail: nori-kkr@umin.ac.jp

Key words: biomarker, angiogenesis, lymphangiogenesis, vascular endothelial growth factor-C, vascular endothelial growth factor-D, vascular endothelial growth factor receptor-3, TMK-1

\section{Introduction}

Surgical resection is the most important treatment for patients with gastric cancer. The prognosis of patients with early gastric cancer after gastrectomy is favorable, with a 5-year survival rate of $\sim 90 \%(1,2)$. The prognosis of patients with advanced gastric cancer remains poor. Even if patients are asymptomatic, the rate of lymph node metastasis and risk of early recurrence is high (3).

Prognostic clinicopathologic factors, including depth of invasion, lymph node status and distant metastasis are important to assess the malignant potential of gastric cancer. Recently, gene expression profiling was developed as a method for defining the phenotypes of malignant tumors at the molecular level. Therefore, indexes based on gene expression may indicate the benefit of systemic therapy and serve as predictive, prognostic and therapeutic biomarkers in gastric cancer (4).

The homeobox (HOX) gene family consists of 39 members with a shared and highly conserved 61 amino acid homeodomain motif. These genes are associated with embryologic regulation, and their roles in neoplastic transformation and tumor progression are now being recognized (5). HOXB9 is a direct transcriptional target of WNT/TCF-4, whose expression is normally restricted to embryogenesis $(5,6)$.

The relationship between HOXB9 expression and tumor malignancy was identified previously. Nguyen et al (7) reported that WNT/TCF signaling through lymphoid enhancer-binding factor 1 (LEF1) and HOXB9 mediated lung adenocarcinoma metastasis. It was reported that elevated HOXB9 expression correlated with high tumor grade in breast cancer patients, and that HOXB9 expression was an independent prognostic factor for disease-free survival $(8,9)$. Fang et al (10) clarified that higher levels of HOXB9 expression were significantly associated with advanced clinical stage in patients with glioma. HOXB9 overexpression also stimulated the proliferation, migration and sphere formation of glioma cells in vitro, and induced the tumorigenicity of glioma cells in vivo.

Induction of HOXB9 in breast cancer cells may increase the concentrations of several angiogenic factors, including vascular endothelial growth factor (VEGF), basic fibroblast growth factor (bFGF) and interleukin (IL)-8. In addition, HOXB9 promotes the formation of large, well-vascularized breast cancer tumors in vivo (8). HOXB9 also induces angiogenesis and tumor proliferation of colon cancer cells in vitro 
resulting in high tumorigenicity in vivo and poor overall survival (11). Bevacizumab, an anti-VEGF antibody, remarkably suppresses tumor proliferation by inhibiting angiogenesis in HOXB9-overexpressing xenografts, improving overall survival and providing prolonged progression-free survival in HOXB9-overexpressing patients (11). These data suggest that HOXB9 has a significant association with tumor progression and therefore may be a prognostic factor in clinical outcomes.

Breast and colon cancer often spread through the blood stream to lung, liver, brain or bones. Regional lymph nodes are the most common site of tumor spread, and lymph node metastasis is a major prognostic factor in gastric cancer (12). Thus, understanding the mechanism of lymphatic metastasis may contribute to the identification of a new therapeutic target for the treatment of gastric cancer. VEGF and its receptors (VEGFRs) have crucial roles in physiological and pathological vasculogenesis. Among VEGFs, VEGF-C and VEGF-D, which bind only to VEGFR-3, are known to regulate lymphangiogenesis (13). These ligands and receptors are often used as lymphangiogenic markers.

In the present study it was hypothesized that, HOXB9 promotes tumor lymphangiogenesis and induces tumor progression, invasion and metastasis in gastric cancer. The aim of this present study was to evaluate the correlation between HOXB9 expression, prognosis and clinicopathologic factors in patients with gastric cancer, and to assess the role of HOXB9 on tumor cell lymphangiogenesis in vitro.

\section{Materials and methods}

Patients. A panel of 58 patients, 42 male and 16 female, with gastric cancer were recruited between June 2008 and February 2012 and were selected from the database from the Department of Surgery of Keio University Hospital (Tokyo, Japan). Their age ranged between 32 and 88 , and the median was 69 years old. The clinical staging was described based on the Union for International Cancer Control TMN Classification of Malignant Tumors (7th edition) (14). The study was approved by the Ethics Committee of Keio University School of Medicine (Tokyo, Japan). Written informed consent was obtained from each patient.

Immunohistochemical staining of HOXB9. Specimens were cut into $4 \mu \mathrm{m}$ sections, mounted onto glass slides, dewaxed in xylene and dehydrated in alcohol. For antigen retrieval, these sections were autoclaved in citric acid solution $(\mathrm{Ph} \mathrm{6.0)}$ at $121^{\circ} \mathrm{C}$ for $10 \mathrm{~min}$. Endogenous peroxidase activity was blocked with a peroxidase blocking reagent $(0.03 \%$ hydrogen peroxide containing sodium azide; Dako; Agilent Technologies, Inc., Santa Clara, CA, USA) for $10 \mathrm{~min}$ at $4^{\circ} \mathrm{C}$. Samples were incubated with a rabbit polyclonal anti-human HOXB9-specific antibody (1:50; cat. no., sc-133671; Santa Cruz Biotechnology, Inc., Dallas, TX, USA) at $4^{\circ} \mathrm{C}$ for $12 \mathrm{~h}$, followed by incubation using a horseradish peroxidase-labeled secondary antibody (cat. no., K4003; Dako; Agilent Technologies, Inc.) at room temperature for $30 \mathrm{~min}$. The slides were stained with 3,3'-diaminobenzidine (Dako; Agilent Technologies, Inc.) at room temperature for $2 \mathrm{~min}$, according to manufacturer's protocol. Slides were rinsed in PBS. Sections were counterstained with hematoxylin, dehydrated and mounted. HOXB9
Table I. Immunoreactive scores of HOXB9.

\begin{tabular}{lr}
\hline Variables & Score \\
\hline Percentage of positive cells & \\
$<33 \%$ & 0 \\
$33-66 \%$ & 1 \\
$>66 \%$ & 2 \\
Intensity of reaction & 0 \\
No reaction & 1 \\
Weak reaction & 2 \\
Intense reaction &
\end{tabular}

0-1 scores $=$ HOXB9 negative, $2-4$ scores $=$ HOXB9 positive. $\mathrm{HOX}$, homeobox.

Table II. Patient characteristics $(n=58)$.

\begin{tabular}{lc}
\hline Parameters & Number $(\%)$ \\
\hline Gender & \\
Male & $42(72.4)$ \\
Female & $16(27.6)$ \\
Median age in years (range) & $69(32-88)$ \\
Pathological depth of tumor invasion (14) & \\
T1 & $14(24.1)$ \\
T2 & $9(15.6)$ \\
T3 & $21(36.2)$ \\
T4 & $14(24.1)$ \\
Pathological nodal stage & \\
N0 & $28(48.3)$ \\
N1 & $8(13.8)$ \\
N2 & $8(13.8)$ \\
N3 & $14(24.1)$ \\
Pathological stage & \\
I & $16(27.6)$ \\
II & $21(36.2)$ \\
II & $19(32.8)$ \\
IV & $2(3.4)$ \\
\hline
\end{tabular}

Pathological tumor stage was described based on the Union for International Cancer Control TMN Classification of Malignant Tumors (7th edition) (14).

expression was evaluated independently by two authors, both blinded to the clinicopathological parameters. A light microscope was used at a magnification of $\mathrm{x} 200$. The percentage of positive cells and staining intensity were evaluated (Table I).

Reverse transcription-quantitative polymerase chain reaction (RT-qPCR). The expression levels of HOXB9 mRNA were determined in four human gastric cancer cell lines (TMK-1, MKN-45, MKN-74 and KATO-III) using RT-qPCR. RNA was extracted from the cells using the RNeasy kit (Qiagen, Inc., 

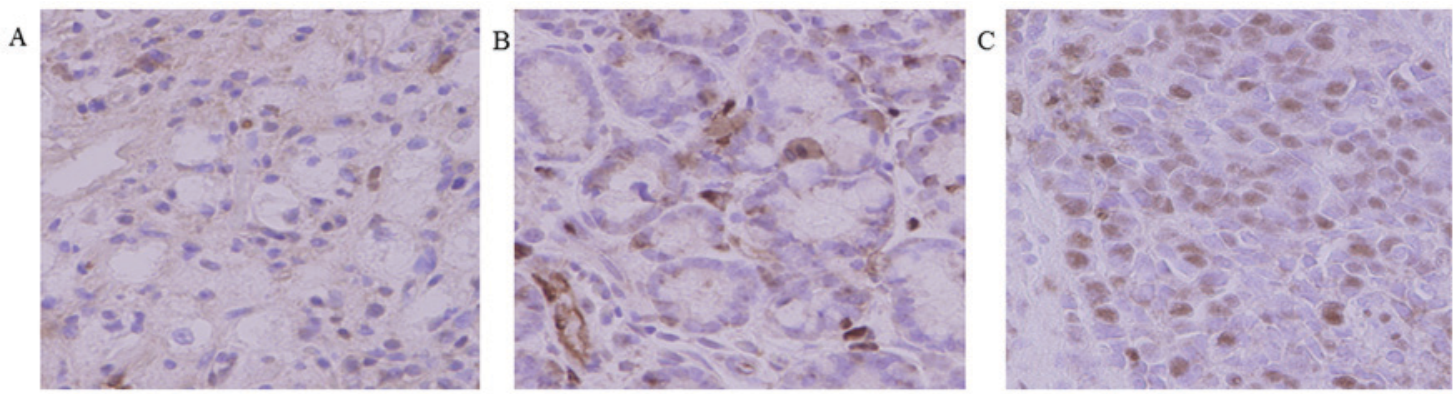

Figure 1. Immunohistochemical staining for homeobox B9 expression in human gastric cancer tissues. Staining intenstity was graded as (A) no reaction, (B) weak reaction and (C) intense reaction (original magnification, $\mathrm{x} 400$ ).

Valencia, CA, USA), according to manufacturer's protocol. The concentration of total RNA was determined using a NanoDrop ND-1000 Spectrophotometer (Thermo Fisher Scientific, Inc., Waltham, MA, USA). Total RNA was reverse transcribed into cDNA using Transcriptor High Fidelity cDNA Synthesis kit (Roche Diagnostics, Rotkreuz, Switzerland) according manufacturer's protocol. The quality and quantity of the cDNA samples were assessed by standard electrophoresis and NanoDrop ND-1000 spectrophotometer. The following primer pairs were used for the qPCR: HOXB9: Sense, 5'-CCG GCTACGGGGACAATAA-3' and antisense, 5'-GGTGTA GGGACAGCGCTTTTT-3'; GAPDH, sense, 5'-ATCATCCCT GCCTCTACTGG-3' and antisense, 5'-TTTCTAGACGGC AGGTCAGGT-3'; and $\beta$-actin: Sense, 5 '-CTCTTCCAGCCT TCCTTCCT-3' and antisense, 5'-AGCACTGTGTTGGCG TACAG-3'. The following thermocycling conditions were used: Initial denaturation at $95^{\circ} \mathrm{C}$ for $2 \mathrm{~min} ; 25$ cycles of $95^{\circ} \mathrm{C}$ for $30 \mathrm{sec} ; 56^{\circ} \mathrm{C}$ for $30 \mathrm{sec}$ and $72^{\circ} \mathrm{C}$ for $60 \mathrm{sec}$; and a final extension at $72^{\circ} \mathrm{C}$ for $10 \mathrm{~min}$. Each experiment was performed using triplicates. qPCR was subsequently performed using a Light Cycler 480 Real-Time PCR System and SYBR Green 1 Master Mix (Roche Diagnostics, Basel, Switzerland) which contained Taq DNA polymerase. The following thermocycling conditions were used for PCR: Pre-denaturation at $95^{\circ} \mathrm{C}$ for $5 \mathrm{~min}$, followed by 45 cycles of denaturation at $95^{\circ} \mathrm{C}$ for $10 \mathrm{sec}$, annealing at $57^{\circ} \mathrm{C}$ for $10 \mathrm{sec}$ and extension at $72^{\circ} \mathrm{C}$ for $10 \mathrm{sec}$. Subsequently, melting curve analysis was performed. The gene expressions were quantified using $2^{-\Delta \Delta C q}$ method (15).

Transfection of HOXB9 gene. TMK-1 cells with low HOXB9 expression were infected with HOXB9-expressing lentiviral vector using pLenti6.3/V5-DEST Gateway Vector kit (Invitrogen; Thermo Fisher Scientific, Inc.), according to the manufacturer's protocols. The time interval between transfection and subsequent experimentation was $>1$ week.

Gel electrophoresis of PCR products. KOD FX Neo (Toyobo Life Science, Osaka, Japan) was used for PCR. The following primer pairs were used: VEGF-C, sense, 5'-AAAGAACCT GCCCCAGAAAT-3' and antisense, 5'-TGGTGGTGGAAC TTCTTTCC-3'; VEGF-D, sense, 5'-TCCAGGAACCAGCTC TCTGT-3' and antisense, 5'-TTTTTGGGGTGCTGGATT AG-3'; and VEGFR-3, sense, 5'-GAGACAAGGACAGCG AGGAC-3' and antisense, 5'-CTGTGTCGTTGGCATGTA CC-3'. The following thermocycling conditions were used: Initial denaturation at $94^{\circ} \mathrm{C}$ for $2 \mathrm{~min}$, followed by 30 cycles at $98^{\circ} \mathrm{C}$ for $10 \mathrm{sec}$ and at $68^{\circ} \mathrm{C} 30 \mathrm{sec}$. Bands were analyzed by electrophoresis in $2 \%$ gel and visualized under UV light. The expression levels of lymphangiogenic factors in HOXB9 transfected cells were compared with that in LacZ transfected cells (control).

$R T$-qPCR for lymphangiogenic markers. RT-qPCR was performed to quantify the difference of expression of lymphangiogenic markers between transfected cells. cDNA purified from the cultured cell lines (TMK-1, TMK-1/LacZ, and TMK-1/HOXB9) were amplified. The thermocycling conditions and fluorophore were the same as described above. The following primer pairs were used: VEGF-D, sense, 5'-GAACAC CAGCACCTCGTACA-3' and antisense; and 5'-TTCTTCAGG GATCTGGATGG-3'; VEGFR-3, sense, 5'-CTGCAAGAACGT GCATCTGT-3' and antisense, 5'-TTCTTGTGGCAGTGCTTG TC-3'. The aforementioned primers were used for VEGF-C. The gene expressions were quantified using $2^{-\Delta \Delta C q}$ method (15).

Statistical analysis. The relevant data were presented as mean \pm standard deviation $(n=3)$. HOXB9 mRNA expression levels in each cell line were compared using the Kruskal-Wallis test. Dunn's multiple comparisons test was used for pairwise post-hoc comparisons. The proportions were compared using Fisher's exact test. Overall survival curves were created by Kaplan-Meier estimates and compared by log-rank tests. Statistical analyses were performed using SPSS (version 19; IBM Corp., Armonk, NY, USA). P $<0.05$ was considered to indicate a statistically significant difference.

\section{Results}

Patient characteristics. Patient characteristics are shown in Table II. The majority of the 58 patients were male (72.4\%) with a median age of 69 years. The median follow-up period was 59.2 months. The majority of the patients (75.9\%) exhibited advanced gastric cancer that had invaded beyond the submucosal layer, and $51.7 \%$ were positive for lymph node metastasis.

HOXB9 expression in patients with gastric cancer. The expression of HOXB9 was evaluated in 58 tumor specimens. Immunohistochemical staining for HOXB9 (Fig. 1) was performed. Staining intenstity was graded as none, weak and intense. According to the classification criteria (Table I), a total of 30 tumors $(51.7 \%)$ were positive for HOXB9 expression. 
Table III. Relation between HOXB9 expression and clinicopathologic factors in 58 gastric cancer patients.

\begin{tabular}{|c|c|c|c|c|}
\hline \multirow[b]{2}{*}{ Characteristics } & \multirow[b]{2}{*}{ No. of patients } & \multicolumn{2}{|c|}{ HOXB9 staining } & \multirow[b]{2}{*}{ P-value } \\
\hline & & Negative $(n=28)$ & Positive ( $\mathrm{n}=30$ ) & \\
\hline Gender & & & & 0.77 \\
\hline Male & 42 & 21 & 21 & \\
\hline Female & 16 & 7 & 9 & \\
\hline Age & & & & 0.19 \\
\hline$<70$ & 29 & 17 & 12 & \\
\hline$\geq 70$ & 29 & 11 & 18 & \\
\hline $\mathrm{T}$ feature & & & & 0.015 \\
\hline T1-2 & 23 & 16 & 7 & \\
\hline T3-4 & 35 & 12 & 23 & \\
\hline LN metastasis & & & & 0.008 \\
\hline Negative & 28 & 19 & 9 & \\
\hline Positive & 30 & 9 & 21 & \\
\hline Lymphatic invasion & & & & 0.001 \\
\hline Negative & 17 & 14 & 3 & \\
\hline Positive & 41 & 14 & 27 & \\
\hline Vascular invasion & & & & 0.023 \\
\hline Negative & 18 & 13 & 5 & \\
\hline Positive & 40 & 15 & 25 & \\
\hline
\end{tabular}

T, pathological depth of tumor invasion; LN: lymph node; HOX, homeobox.

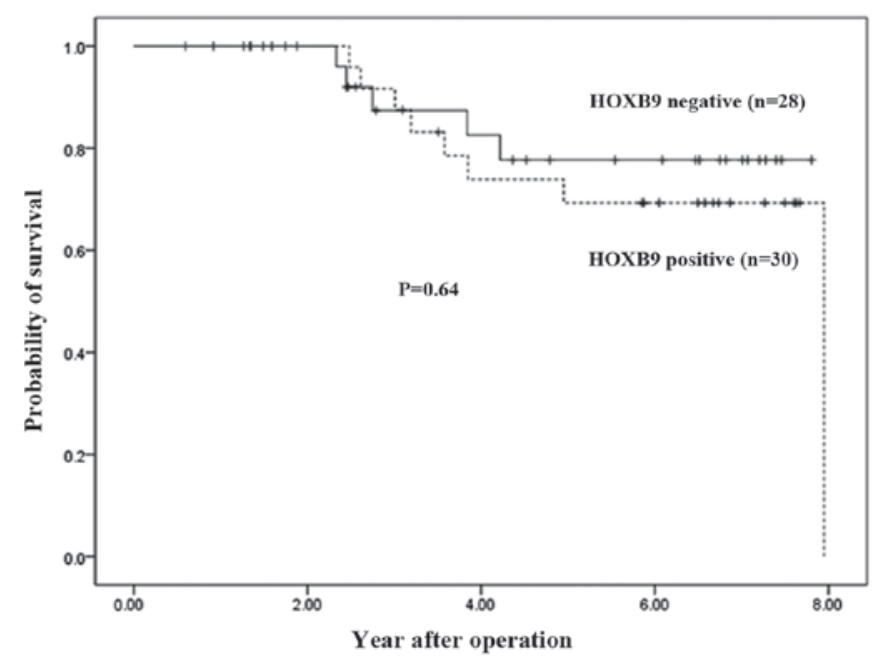

Figure 2. Kaplan-Meier plots of overall survival in HOXB9-positive and HOXB9-negative patients with gastric cancer. The $\mathrm{P}$ value was calculated by the log-rank test. HOX, homeobox.

HOXB9 staining was observed in the nucleus of gastric cancer cells. Association between HOXB9 expression and clinicopathologic variables is shown in Table III.

In addition, overall survival was decreased in patients with positive HOXB9 expression ( $\mathrm{P}=0.64$; Fig. 2). However, this did not reach a statistically significant difference. The depth of tumor invasion $(\mathrm{P}=0.015)$, the number of node metastases $(\mathrm{P}=0.008)$, lymphatic invasion $(\mathrm{P}=0.001)$ and vascular invasion ( $\mathrm{P}=0.023)$ were significantly associated with HOXB9 expression (Table III).

Association between HOXB9 and lymphangiogenic factors. TMK-1 cells expressed the lowest amount of HOXB9 mRNA expression levels among the four cell lines tested (Fig. 3). Using a viral vector, HOXB9 mRNA expression level was increased by 3,377-fold in TMK-1/HOXB9 cells compared with that in TMK-1/LacZ cells; this difference reached statistical significance ( $\mathrm{P}=0.027$; Fig. 4). Gel electrophoresis of PCR products showed that the expression levels of VEGF-D mRNA were increased in TMK-1/HOXB9 cells compared with TMK-1/LacZ cells (Fig. 5). The relative mRNA level of VEGF-D was increased by 6.06-fold in TMK-1/HOXB9 compared with that in TMK-1/LacZ cells ( $\mathrm{P}=0.066$; Fig. 6). VEGF-C and VEGFR-3 levels were unchanged (Figs. 5 and 6).

\section{Discussion}

HOXB9 is considered to be a transcription factor. Transcription factors are proteins, which bind to DNA sequences to control the expression of genes (16). They may have a dynamic behavior depending on intracellular/extracellular signals. In the present study, the expression of HOXB9 and clinicopathological factors were investigated in patients with gastric cancer at various pathological stages, in order to compare the association between HOXB9 expression and gastric cancer progression. Immunohistochemistry revealed that $51.7 \%$ of resected gastric cancer tissues were positive for HOXB9 expression. In a previous 


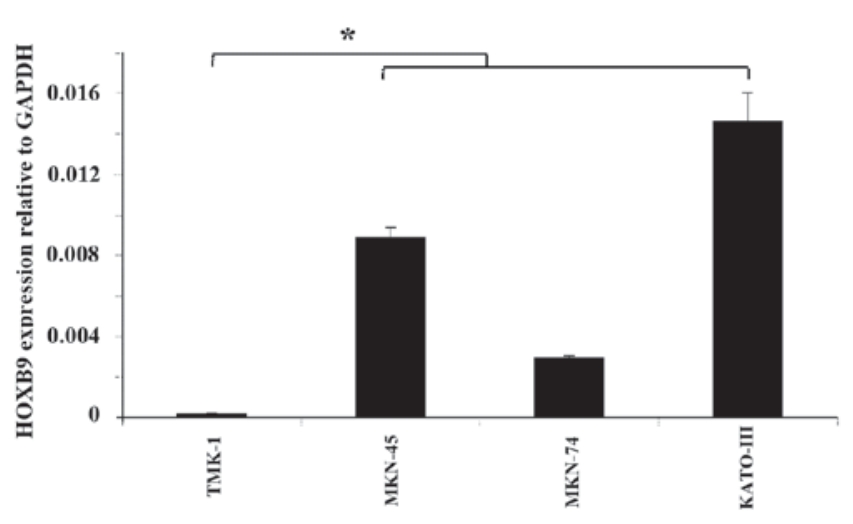

Figure 3. Comparison of HOXB9 mRNA expression levels in in TMK-1, MKN-45, MKN-74 and KATO-III cell lines. TMK-1 cells exhibited the lowest HOXB9 expression level among all cell lines. ${ }^{*} \mathrm{P}=0.083$ with comparisons as indicated by lines. HOX, homeobox.

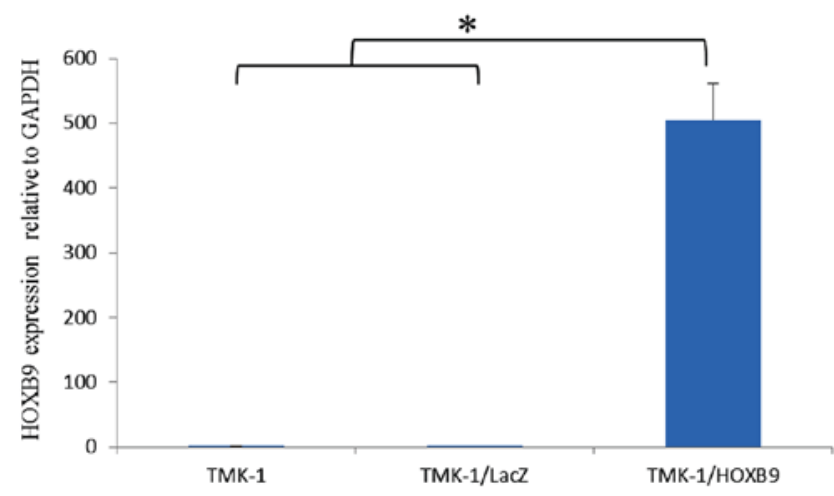

Figure 4. Comparison of HOXB9 mRNA expression levels in TMK-1, TMK-1/LacZ and TMK-1/HOXB9 in cell lines. Overexpression of HOXB9 mRNA in TMK-1 cells. "P<0.05 with comparisons indicated by lines. HOX, homeobox.

study, it was showed that HOXB9 was expressed in $49 \%$ of resected breast cancer tissues and in $67 \%$ of resected colorectal cancer tissues $(9,11)$. Although the methods used to evaluate HOXB9 expression were different in each study, the positive rates were comparable. In addition, it was indicated that HOXB9 expression was localized to the nucleus of gastric cancer cells, which is in accordance to a previous study on breast cancer (9).

In the present study, HOXB9-positive patients with gastric cancer tended to exhibit a poorer prognosis compared with those in the HOXB9-negative group. Although this difference did not reach statistical significance, it is possible that additional data may clarify the potential association between HOXB9 expression and survival of patients with gastric cancer. HOXB9 expression was the only independent prognostic factor for disease-free survival in breast cancer patients (hazard ratio $=15.532 ; \mathrm{P}=0.009)(9)$, and increased HOXB9 expression in colorectal cancer was significantly associated with poorer overall survival (11). However, Sha et al (17) reported that decreased expression of HOXB9 was associated with a poor overall survival in Chinese patients with gastric cancer. The reason for this discrepancy in the association between survival and HOXB9 expression are unclear, but may be related to differences in the subjects and methodology.

In our previous study, HOXB9 positivity was significantly associated with tumor virulence in breast cancer patients

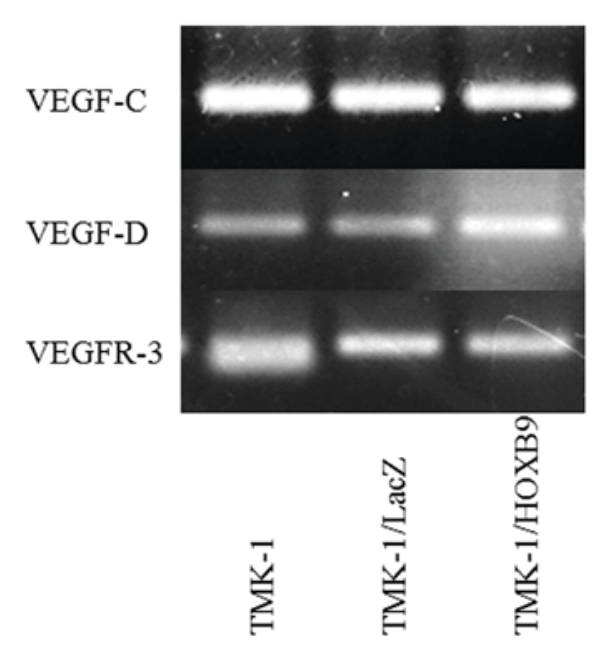

Figure 5. Expression levels of VEGF-C, VEGF-D and VEGFR-3 mRNA in TMK-1, TMK-1/LacZ and TMK-1/HOXB9 cells. The expression of VEGF-D mRNA was increased in TMK-1/HOXB9 compared with TMK-1/LacZ. VEGF-C and VEGFR-3 levels were unchanged. HOX, homeobox; VEGF, vascular endothelial growth factor; VEGFR, VEFG receptor.

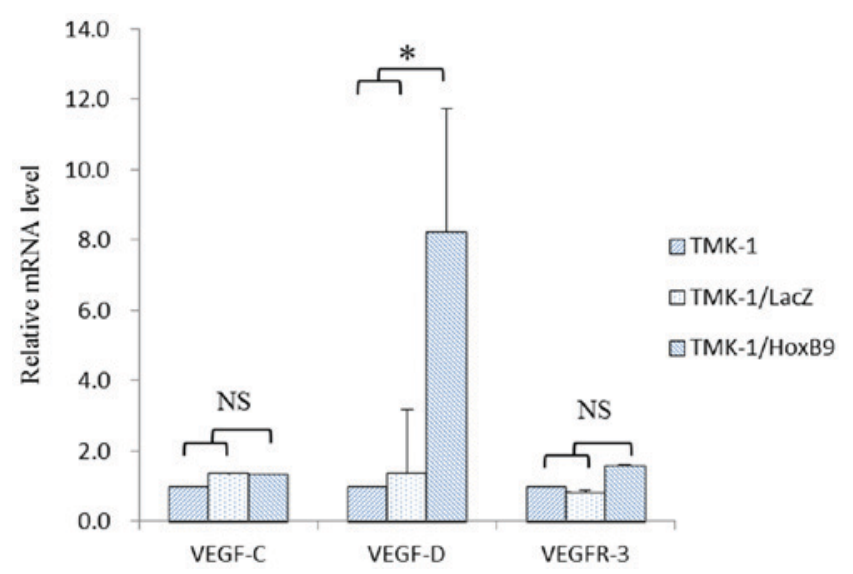

Figure 6. Quantification of expression levels of lymphangiogenic markers. mRNA expression levels of lymphangiogenic factors in TMK-1, TMK-1/LacZ and TMK-1/HOXB9 cell lines were compared. The relative mRNA level of VEGF-D was increased by 6.06 -fold in TMK-1/HOXB9 cells compared with that in TMK-1/LacZ cells. " $\mathrm{P}=0.066$ with comparisons indicated by lines. HOX, homeobox; VEGF, vascular endothelial growth factor; VEGFR, VEFG receptor; NS, not significant.

(tumor size, nuclear grade and lymph node metastasis) (9). In the present study, it was found that HOXB9 expression was significantly associated with the depth of invasion, lymph node metastasis, lymphatic invasion and vascular invasion in patients with gastric cancer, suggesting a role of this transcription factor in gastric cancer.

Lymph node metastasis and lymphatic invasion are related to lymphogenic metastasis, a specific phenomenon of gastric cancer progression (12). A previous study reported that HOXB9 induces the expression of several angiogenic factors (epidermal growth factor (EGF), bFGF, IL-8 and angiopoietin-like 2 (ANGPTL-2)), as well as ErbB (amphiregulin, epiregulin and neuregulins) and transforming growth factor- $\beta$ (TGF- $\beta$ ) in patients with breast cancer (8). These factors activate their respective pathways, leading to increased cell motility and the acquisition of mesenchymal phenotypes (8). Additionally, a 
study on colon cancer reported that HOXB9 induced angiogenesis and tumor proliferation in vitro, consistent with high tumorigenicity in vivo (11). The results of the present study demonstrated that there was no association between HOXB9 expression and the angiogenic factors VEGF-A, bFGF, IL8, ANGPTL2, TGF- $\beta 1$, and TGF- $\beta 2$ in patients with gastric cancer (data not shown). However, the lymphangiogenic factor VEGF-D, but not VEGF-C and VEGFR-3, was elevated in TMK-1 cells transfected with the HOXB9 gene. To the best of our knowledge this is the first study suggesting that HOXB9 promotes lymphangiogenesis. Therefore, HOXB9 may be associated with lymphogenic metastasis.

Overall, HOXB9 expression positively associated with gastric cancer progression and may be associated with poor prognosis. These results suggest the potential clinical utility of HOXB9 as a prognostic factor or therapeutic target in gastric cancer.

\section{Acknowledgements}

The authors would like to thank Dr. Y. Hoshino and Dr. H. Seki (Department of Surgery, Keio University School of Medicine) for the help with designing these experiments. The authors would also like to thank Mrs. Y. Yoshimura, Mr. H. Okazaki, Mr. K. Miyao and Mrs. S. Fukuhara (Department of Surgery, Keio University School of Medicine) for their technical assistance with all aspects of the present study.

\section{Funding}

The present work was supported by a JSPS KAKENHI Grant-in-Aid for Young Scientists (B) (grant no. 26861103) and a Grant-in-Aid for Scientific Research (C) (grant nos. 22591469 and 25462037).

\section{Availability of data and materials}

The datasets used and/or analyzed during the present study are available from the corresponding author on reasonable request.

\section{Authors' contributions}

FK, NW, TH, and YK conceived the study. RN, TT, HK, and HT were the major contributors in analyzing and interpreting the patient data. FK and KF performed the experiments. FK and NW evaluated immunostained sections. FK wrote the manuscript. YK supervised the study, and performed the critical review. All authors read and approved the final manuscript.

\section{Ethics approval and consent to participate}

The study was approved by the Ethics Committee of Keio University School of Medicine (approval no. 20080189). Written informed consent was obtained from each patient.

\section{Patient consent for publication}

The patients consented to publication.

\section{Competing interests}

The authors declare that they have no competing interests.

\section{References}

1. Sano T, Sasako M, Kinoshita T and Maruyama K: Recurrence of early gastric cancer. Follow-up of 1475 patients and review of the Japanese literature. Cancer 72: 3174-3178, 1993.

2. Japanese Gastric Cancer Association Registration Committee, Maruyama K, Kaminishi M, Hayashi K, Isobe Y, Honda I, Katai H, Arai K, Kodera Y and Nashimoto A: Gastric cancer treated in 1991 in Japan: Data analysis of nationwide registry. Gastric Cancer 9: 51-66, 2006.

3. Gotoda T, Yanagisawa A, Sasako M, Ono H, Nakanishi Y, Shimoda T and Kato Y: Incidence of lymph node metastasis from early gastric cancer: Estimation with a large number of cases at two large centers. Gastric Cancer 3: 219-225, 2000.

4. Bang YJ, Van Cutsem E, Feyereislova A, Chung HC, Shen L, Sawaki A, Lordick F, Ohtsu A, Omuro Y, Satoh T, et al: Trastuzumab in combination with chemotherapy versus chemotherapy alone for treatment of HER2-positive advanced gastric or gastro-oesophageal junction cancer (ToGA): A phase 3, open-label, randomised controlled trial. Lancet 376: 687-697, 2010.

5. Abate-Shen C: Deregulated homeobox gene expression in cancer: Cause or consequence? Nat Rev Cancer 2: 777-785, 2002.

6. Hatzis P, van der Flier LG, van Driel MA, Guryev V, Nielsen F, Denissov S, Nijman IJ, Koster J, Santo EE, Welboren W, et al: Genome-wide pattern of TCF7L2/TCF4 chromatin occupancy in colorectal cancer cells. Mol Cell Biol 28: 2732-2744, 2008.

7. Nguyen DX, Chiang AC, Zhang XH, Kim JY, Kris MG, Ladanyi M, Gerald WL and Massagué J: WNT/TCF signaling through LEF1 and HOXB9 mediates lung adenocarcinoma metastasis. Cell 138: 51-62, 2009.

8. Hayashida T, Takahashi F, Chiba N, Brachtel E, Takahashi M, Godin-Heymann N, Gross KW, Vivanco Md, Wijendran V, Shioda T, et al: HOXB9, a gene overexpressed in breast cancer, promotes tumorigenicity and lung metastasis. Proc Natl Acad Sci USA 107: 1100-1105, 2010.

9. Seki H, Hayashida T, Jinno H, Hirose S, Sakata M, Takahashi M, Maheswaran S, Mukai M and Kitagawa Y: HOXB9 expression promoting tumor cell proliferation and angiogenesis is associated with clinical outcomes in breast cancer patients. Ann Surg Oncol 19: 1831-1840, 2012.

10. Fang L, Xu Y and Zou L: Overexpressed homeobox B9 regulates oncogenic activities by transforming growth factor- $\beta 1$ in gliomas. Biochem Biophys Res Commun 446: 272-279, 2014.

11. Hoshino Y, Hayashida T, Hirata A, Takahashi H, Chiba N, Ohmura M, Wakui M, Jinno H, Hasegawa H, Maheswaran S, et al: Bevacizumab terminates homeobox B9-induced tumor proliferation by silencing microenvironmental communication. Mol Cancer 13: 102, 2014.

12. Pak KH, Jo A, Choi HJ, Choi Y, Kim H, and Cheong JH: The different role of intratumoral and peritumoral lymphangiogenesis in gastric cancer progression and prognosis. BMC Cancer 15: 498, 2015.

13. Takahashi S: Vascular endothelial growth factor (VEGF), VEGF receptors and their inhibitors for antiangiogenic tumor therapy. Biol Pharm Bull 34: 1785-1788, 2011.

14. Sobin LH, Gospodarowicz MK and Wittekind C: TNM classification of malignant tumours. 7th edition. Wiley-Blackwell, Hoboken, Oxford, 2009.

15. Livak KJ and Schmittgen TD: Analysis of relative gene expression data using real-time quantitative PCR and the 2(-Delta Delta C(T)) method. Methods 25: 402-408, 2001.

16. Bouhlel MA, Lambert M and David-Cordonnier MH: Targeting transcription factor binding to DNA by competing with DNA binders as an approach for controlling gene expression. Curr Top Med Chem 15: 1323-1358, 2015.

17. Sha S, Gu Y, Xu B, Hu H, Yang Y, Kong X and Wu K: Decreased expression of HOXB9 is related to poor overall survival in patients with gastric carcinoma. Dig Liver Dis 45: 422-429, 2013. 